\title{
Improvements in image analysis and fluorescence microscopy to discriminate and enumerate bacteria and viruses in aquatic samples
}

\author{
Alexander Shopov, Samanthia C. Williams, Peter G. Verity* \\ Skidaway Institute of Oceanography, 10 Ocean Science Circle, Savannah, Georgia 31411, USA
}

\begin{abstract}
Commercial image analysis software includes functions for image filtering and segmentation, but few packages contain automated cell counting routines. Software capable of performing object enumeration functions adequately when samples contain brightly illuminated particles on a dark, even background. However, although current sample preparation protocols for aquatic bacteria and virus samples conceptually yield images ideal for such analysis, the reality can be quite different. Coastal and estuarine waters, in particular, contain considerable particulate organic matter which, when stained for epifluorescent microscopy, produces images incorporating numerous particles of different size, color, and optical density. We have modified an earlier automated image analysis protocol, in order to discriminate and analyze suspended cells based on size and optical density parameters, as well as cells associated with detrital matter. For the enumeration of free bacteria versus bacteria attached to detritus, this approach employs a color segmentation algorithm to create an alpha-channel mask of the detritus. This mask can then be used as a template over the original image, permitting enumeration of both free bacteria and attached bacteria. This software protocol can be used in conjunction with specific staining protocols to evaluate relationships among bacteria, viruses, and detritus in aquatic samples.
\end{abstract}

KEY WORDS: Image analysis $\cdot$ Bacteria $\cdot$ Viruses $\cdot$ Detritus $\cdot$ Microscopy

Resale or republication not permitted without written consent of the publisher

\section{INTRODUCTION}

Enumeration of bacterial and viral cells is essential to understanding the role they play in diverse aquatic environments. Over the years, many methods have been developed to enumerate microscopic marine organisms, of which epifluorescent direct count techniques have emerged as the method of choice (Kepner \& Pratt 1994). There are, however, several limitations associated with such techniques. For example, manual enumeration of cells is highly labor intensive and can suffer from investigator bias.

Image analysis was first applied to enumeration of aquatic bacteria 2 decades ago (Krambeck et al. 1981) and, coupled with epifluorescence microscopy, has

*Corresponding author. E-mail: peter@skio.peachnet.edu become a premier quantitative tool in deconvolving the important roles of bacterioplankton (Sieracki et al. 1985, Bjørnsen 1986, Schroder \& Krambeck 1991, Verity \& Sieracki 1993).

Digital image processing software typically includes functions for spatial filtering and image segmentation. Some commercial packages are also capable of performing particle enumeration within specific opticaldensity ranges, provided the images contain bright cells on a dark, evenly lit background. However, these criteria are not always met in images of fluorescently stained natural populations. The edge-enhancement and object detection protocol developed by Viles \& Sieracki (1991) eliminated many of the technical problems found in images of epifluorescently stained samples. Their method was optimized for processors available at the time of its development, but recent developments in processor power and greater system 
resources permit modifications to the Viles \& Sieracki (1991) method, which allows for a more efficient segmentation technique. One such automated segmentation and counting procedure is described here.

Once an accurate system of cell enumeration was implemented, the subsequent goal was development of a protocol to differentiate between attached and unattached bacteria in samples that contained large amounts of detrital matter. Combining scientific and artistic digital imaging expertise with existing sample preparation and capturing protocols, a method was developed whereby an alpha-channel (grayscale opacity map of an image used in digital compositing, in which gray values represent varying levels of transparency within the image) mask of the detritus was created for each image, and then reapplied to the image after the new segmentation procedures were implemented. Enumeration was then conducted on both attached and unattached bacteria in each image.

\section{METHODS}

The imaging system used a desktop PC with a $400 \mathrm{MHz}$ Pentium II MMX processor, $128 \mathrm{MB}$ of RAM, and the Windows 98 operating system. Images were visualized through an Olympus BX 60 epifluorescent microscope equipped with a $100 \times$ UPLANFL NA 1.3 oil objective. The images were then captured by a Photonic Science Color Cool View cooled three-chip color integrating Charge-Coupled Device (CCD) camera. Image-Pro Plus v3.0.1 was used to capture $768 \times 576$ TIFF images, at a color depth of 8 bits ( 256 colors, with 0 as black and 255 as white) per pixel. Bacterial samples were stained with the Vital Stain Probe (VSP) method (Williams et al. 1998). The VSP method yields cells stained with DAPI (excitation [ex] 437, emission [em] 490), propidium iodide (PI: ex 505, em 615), and TRITC(ex 544, em 572), FITC- (ex 460, em 515), or CY3(ex 550, em 570) labelled 16S rRNA targeted oligonucleotide probes. Virus samples were stained with SYBR Gold (ex 495, em 537) (Lu et al. 1999), and images captured through an Omega Optical FITC filter set (XF 23), with exciter 485 DF22 and emitter 535 DF35 filter.

Image analysis was controlled by an automated imaging system, 'Skidaway Tools' ${ }^{1}$, developed using Microsoft's Visual Basic 6.0 and Visual C++ 5.0 develop-

\footnotetext{
${ }^{1}$ The 'Skidaway Tools' protocol and documentation is available to download as a .zip archive via the lab Website located at http://www.skio.peachnet.edu/faculty/verity/downloads.html, or on floppy disk via regular mail at 'Skidaway Tools', c/o Dr Peter G. Verity, Skidaway Institute of Oceanography, 10 Ocean Science Circle, Savannah, Georgia 31411, USA. The 'Skidaway Tools' source code will remain proprietary
}

ment environments. This system is programmed to interface directly with Media Cybernetics Image-Pro Plus v3.0.1 and its scripting language Auto-Pro, thereby using Image-Pro Plus as an enumeration and data analysis workhorse, while all of the enhancement and segmentation routines operate through 'Skidaway Tools'. The complete 'Skidaway Tools' event loop is shown in Fig. 1.

'Skidaway Tools' was developed using formulas presented by Viles \& Sieracki (1991), based on the MarrHildreth Gaussian-smoothed Laplacian edge-detection protocol (Marr \& Hildreth 1980), with added flat-fielding and edge-strength operators. Flat fielding is the process of removing signal noise from an image. The Viles \& Sieracki (1991) flat-fielding operator used a calibration and bias image to eliminate background noise and unbalanced lighting gradients present in the image. Here, 'Skidaway Tools' relied on an alternative background subtraction algorithm (Gauthier et al. 1992) requiring only a calibration image, captured by moving the microscope stage to an area of the filter devoid of any objects and taking an image of just the background fluorescence. This algorithm calculated the difference in intensity of a given pixel in the calibration image from the mean pixel intensity of the overall calibration image (Fig. 2A). This value was then added to

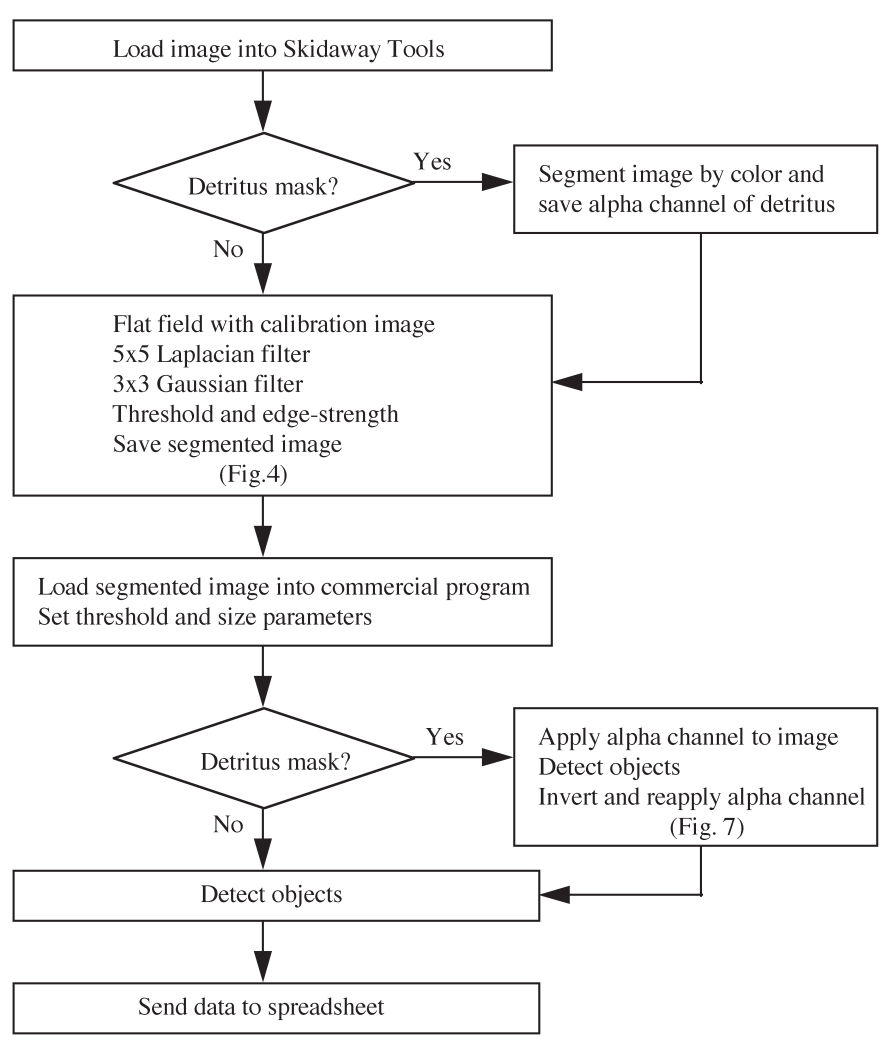

Fig. 1. Flow chart of the 'Skidaway Tools' event loop. See 'Results' and 'Discussion' for details 
each pixel of the input image, $I_{i j}$, using the following algorithm:

$$
I_{i j}^{c}=I_{i j}+\left(\bar{b}-b_{i j}\right)
$$

where $I_{i j}^{C}$ is the calibrated intensity of the original pixel, and $\bar{b}$ is the average intensity of all the pixels of the calibration image $b_{i j}$. The input image with the flatfielding process applied is shown in Fig. 2A.

An additional modification was the substitution of the $3 \times 3$ Laplacian Kernel

$$
\begin{array}{rrr}
0 & -1 & 0 \\
-1 & 4 & -1 \\
0 & -1 & 0
\end{array}
$$

with a variation of a $5 \times 5$ Laplacian Kernel:

$$
\begin{array}{rrrrr}
0 & 0 & -1 & 0 & 0 \\
0 & -1 & 1 & -1 & 0 \\
-1 & 1 & 4 & 1 & -1 \\
0 & -1 & 1 & -1 & 0 \\
0 & 0 & -1 & 0 & 0
\end{array}
$$

This modified Kernel was applied to each pixel in the input image, resulting in an enhancement of the cell boundaries (Fig. 2B). Application of the Gaussian Kernel (Fig. 2C) removes any image noise generated by the Laplacian operator. The threshold and edgestrength operators were then applied to each pixel of the filtered image as described by Viles \& Sieracki (1991), producing a bilevel image in which accepted pixels were set to white and all other pixels set to black (Fig. 2D)

To discriminate bacteria embedded in detritus from free bacteria, the use of an alpha-channel mask was developed, whereby in the commercial software the user segmented the image via color differences between detritus and living cells (Williams et al. 1995). Pixels corresponding to detritus were set to black, while the remaining pixels were saturated to pure white. This segmented image was then saved as a separate file. The mask was then applied to the input image, which had already been filtered and segmented by the above method. Using a reverse of the traditional alpha-channel (whereby the white pixels are given an opacity of $100 \%$ and the black pixels $0 \%$ ), the opacity of the white pixels was instead set to $0 \%$, covering the detritus with the black, $100 \%$ opaque regions
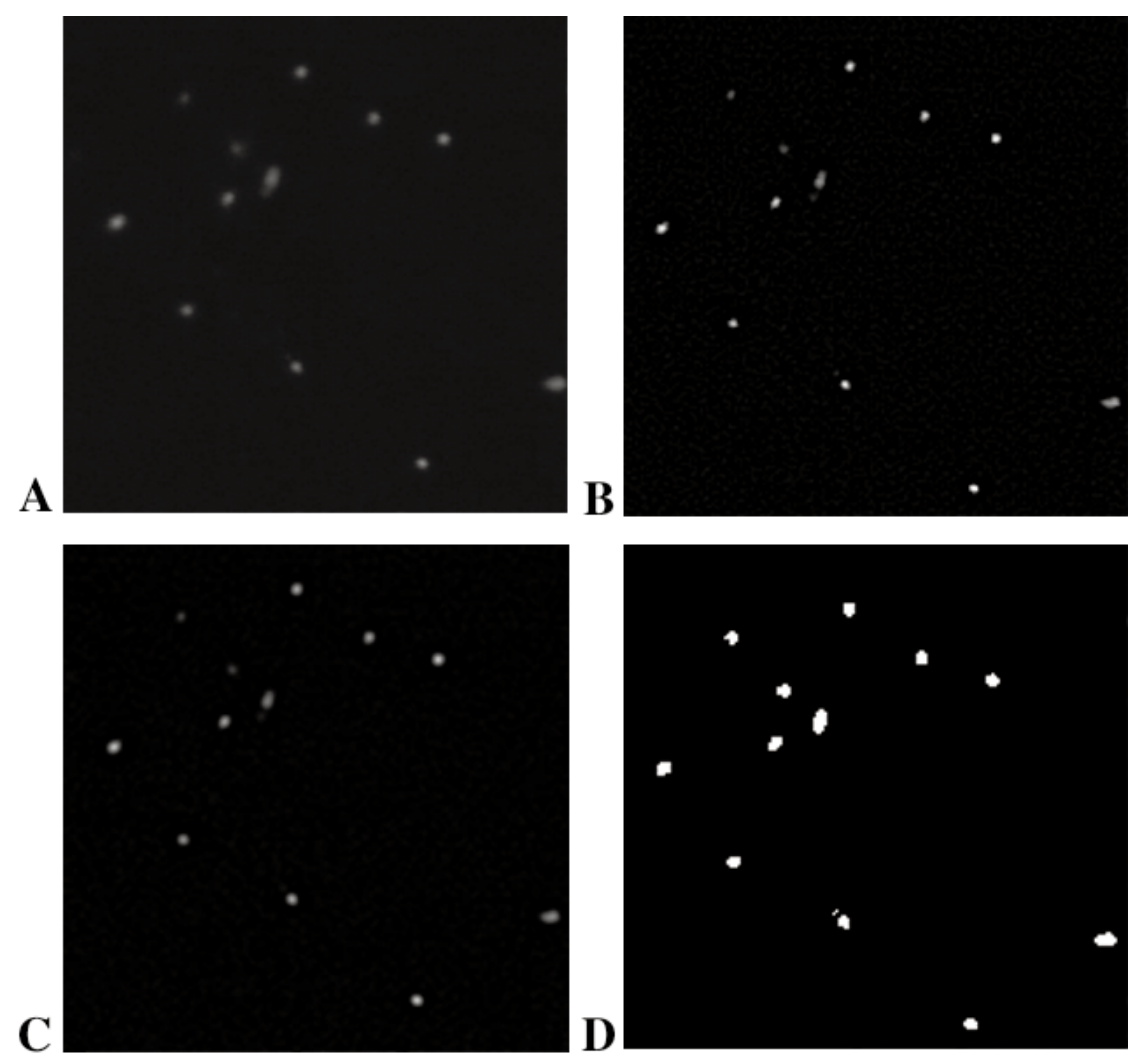

Fig. 2. (A) Bacteria stained with DAPI produce images ideally suited for automated image analysis (i.e. bright cells on a dark, even background). By applying the flat-fielding operator, camera signal noise is eliminated from the image. (B) Modified $5 \times 5$ Laplacian filter enhances cell edges. (C) $3 \times 3$ Gaussian filter removes background noise generated by the Laplacian operation. (D) Threshold and edge-strength parameters are applied to the filtered image providing an accurate representation of the cell boundaries 
of the mask, while leaving the background of the image visible. The resulting image of only free bacteria was then enumerated. The mask was subsequently inverted and reapplied to the original input image. The result was the background covered by the mask, leaving only the detrital matter visible. This image was then also counted, yielding the number of bacteria associated with the detritus. The edge strength operator eliminated the residual detritus left by the segmentation procedure.

'Skidaway Tools' provides functions for image segmentation and detrital masking only. With the ability of Image-Pro Plus to provide counts based on a global threshold value, images segmented and filtered by 'Skidaway Tools' were piped to the commercial software for enumeration. As 'Skidaway Tools' produced black and white bilevel images, Image-Pro Plus performed an enumeration of the image using a global threshold value of 255, and appropriate size parameters based on known size-ranges of fluorescing bacterial and viral cells. Counts were sent back to 'Skidaway Tools', where the information was processed and all calculations were written into spreadsheets.

\section{RESULTS AND DISCUSSION}

Images of samples consisting of fluorescently stained cells tend to contain uneven areas of light and dark intensities (Fig. 3A). A major problem inherent in threshold-based segmentation algorithms is the inability to deal with an unbalanced image histogram (Fig. 3B), usually the result of background noise and uneven lighting. As most commercial software utilizes image histograms for object segmentation, the automated enumeration of fluorescently stained bacterial or viral cells becomes inherently difficult, requiring tedious and time-consuming direct manual counts in order to achieve acceptable accuracy. Otherwise, the uneven background causes threshold values to segment the cells within the image's hotspot at the expense of cells located within the darker regions (Fig. 3C). Likewise, threshold values required to segment cells within the darker regions of the image cause the high-intensity area of the image to be segmented as a single object (Fig. 3D). When the same images are filtered using the protocol described here (Fig. 4A), the image histogram is balanced (Fig. 4B), showing a consistent background
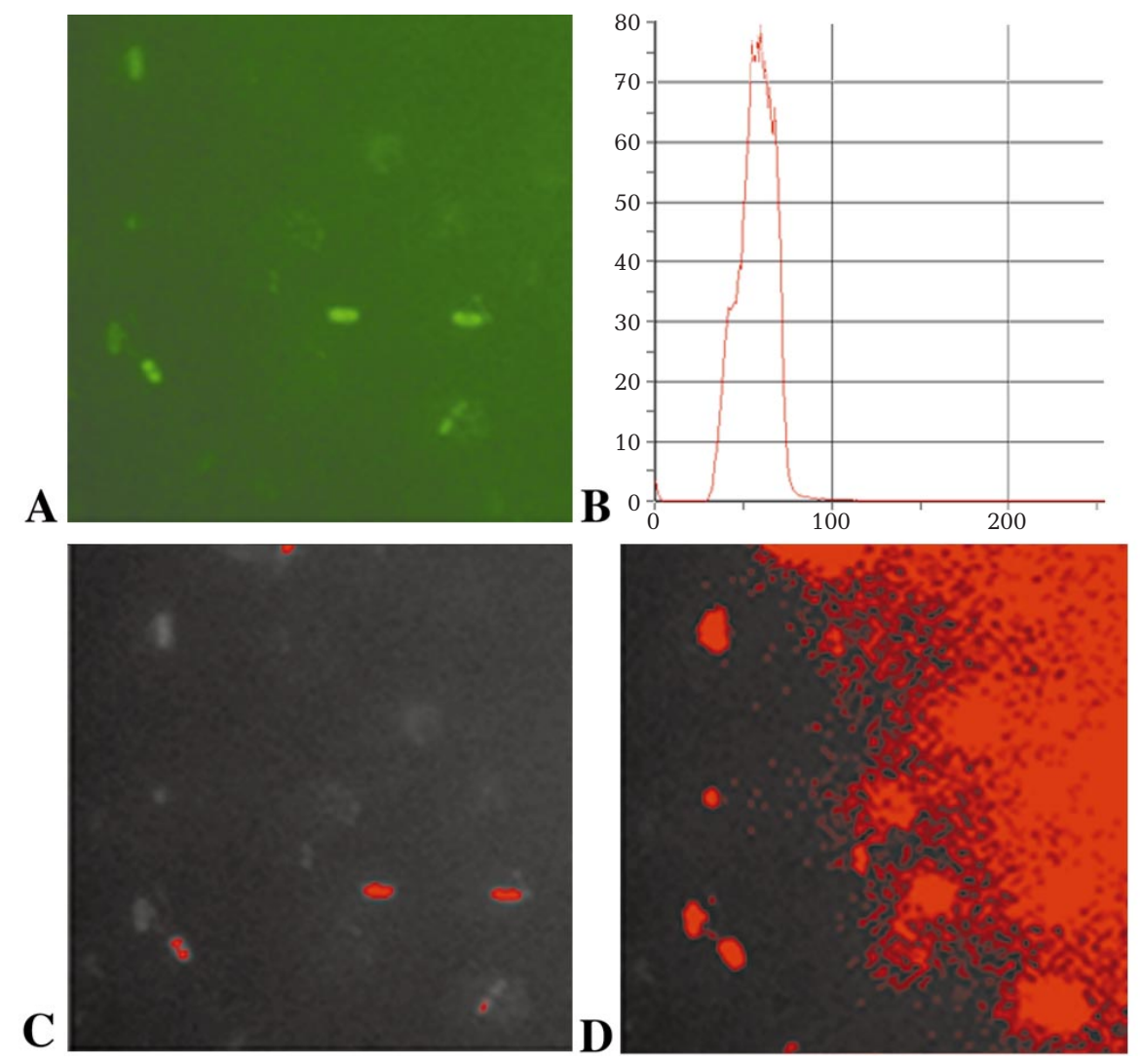

Fig. 3. (A) Bacteria treated with FITC-labelled 16S rRNA oligonucleotide probes according to Williams et al. (1998). (B) Intensity histogram of image (A). (C,D) Use of commercially available image analysis software to globally threshold a fluorescent image which contains an uneven background: either cells composed of dim pixels are missed (C) or bright pixels are incorrectly recognized (D) 

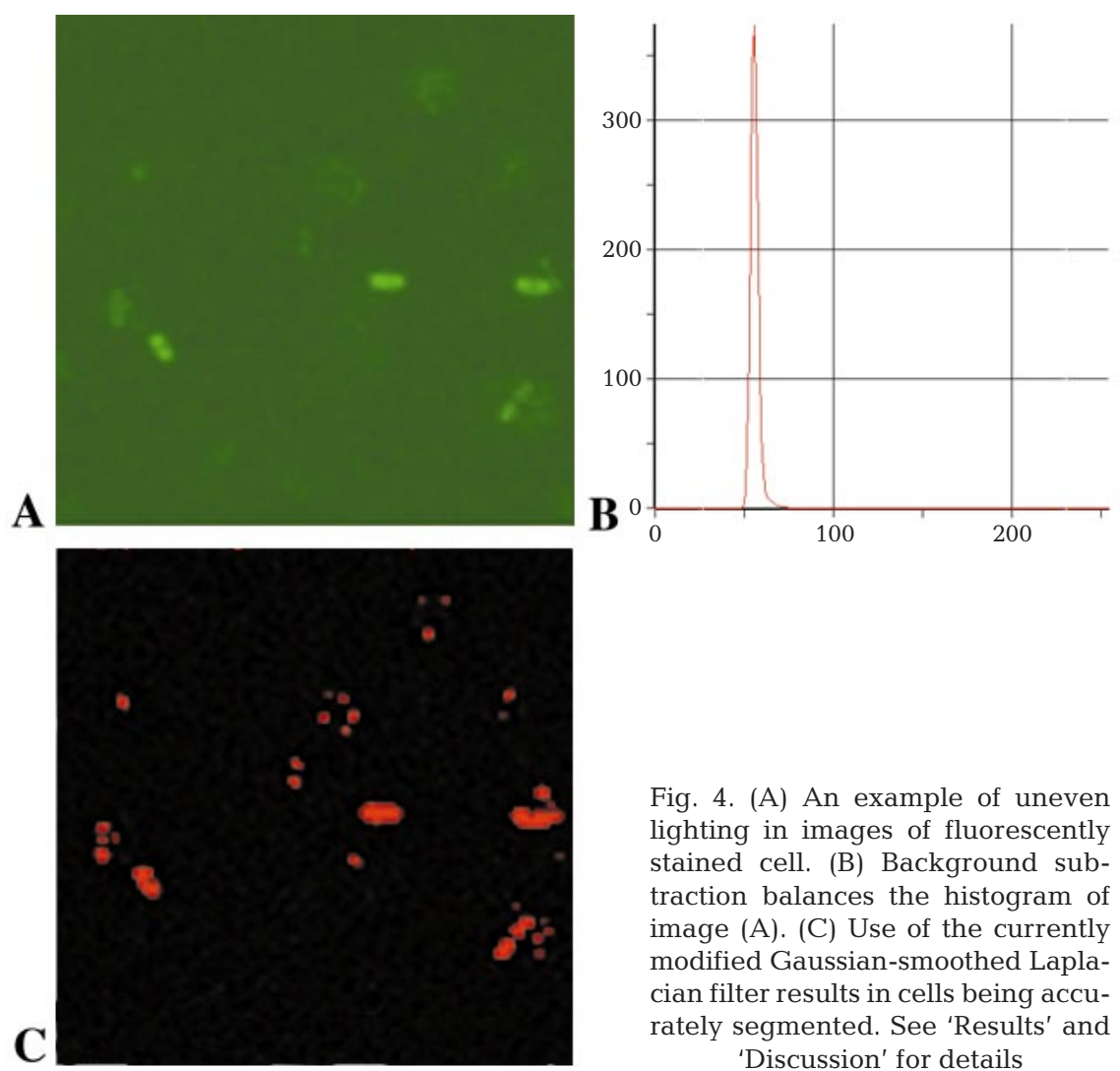

Fig. 4. (A) An example of uneven lighting in images of fluorescently stained cell. (B) Background subtraction balances the histogram of image (A). (C) Use of the currently modified Gaussian-smoothed Laplacian filter results in cells being accurately segmented. See 'Results' and 'Discussion' for details on a dark background. However, images of bacteria cells stained with TRITC and FITC 16S rRNA oligonucleotide probes and viruses stained with SYBR Gold tended to display cells that fluoresced with an intensity only slightly greater than that of the background. The $3 \times 3$ Laplacian filter, as originally described by Viles \& Sieracki (1991) and also utilized by Blackburn et al. (1998), performed well with the brighter bacteria cells in the DAPI- and PI-stained images, but often removed probe- and SYBR Gold-stained cells (Fig. 5A) as part of the background (Fig. 5B). The $5 \times 5$ Laplacian filter, however, was able to pick out the edges of these objects so they would not be overlooked by the edge-strength operator (Fig. 5C).

The image segmentation protocol resulted in black and white bilevel images in which segmented cells were white and the image background was black. The commercial software was able to intensity, and allowing cell boundaries to be accurately segmented (Fig. 4C).

With the possible exception of DAPI, fluorescent stains can often produce images with areas of uneven intensity. Flat-fielding is a technique by which the overall intensity of an unbalanced image is equalized and background noise filtered out, thereby balancing the image histogram. This is typically accomplished through the use of background calibration images, captured from areas of the sample filter that do not contain any cells or other objects, or by using bias images, which are obtained with the camera shutter closed and consist of signal noise generated by the camera. This combination of bias and calibration operators accurately removes both unbalanced lighting and residual camera noise from a digital image. Although the flat-fielding operator provided by Viles \& Sieracki (1991) performed well, the Photonic Science camera used here did not generate significant signal noise; therefore bias-image computations were unnecessary. Gauthier et al. (1992) described a flat-fielding operator requiring only a single calibration image, and that was computationally less expensive than the Viles \& Sieracki (1991) algorithm. This equation performed accurately with each of the staining protocols used.

Using the VSP protocol, images captured of DAPIand PI-stained cells typically consisted of bright cells enumerate these images using a global threshold value of 255. Samples enumerated manually and samples segmented by 'Skidaway Tools' yielded statistically similar cell abundances (Fig. 6). The slope of the linear regression was not significantly different from 1 $(p<0.0001)$, and the intercept was not significantly different from 0 ( $p<0.0001)$. Thus, 'Skidaway Tools' segmentation algorithm, when combined with commercial software, provided accurate, rapid, and fully automated cell segmentation and enumeration.

It has been suggested previously that bacteria associated with detrital matter (Fig. 7A) could be enumerated by capturing the image through a bandpass filter, thereby eliminating the detritus in the final image (Viles \& Sieracki 1991). Here the $5 \times 5$ Laplacian filter was used for the same purpose as the bandpass filter, filtering out the detritus which had an intensity range greater than the background but less than the cells and enhancing the edges of the bacterial cells, thereby allowing all cells to be discriminated (Fig. 7B). The concept of using an alpha-channel (Fig. 7C) evolved from the need to acquire separate counts for free bacteria (Fig. 7D) and attached bacteria (Fig. 7E). By applying the mask to the images, both cell types were enumerated.

As a baseline solution for general digital image analysis, commercial software packages perform image 

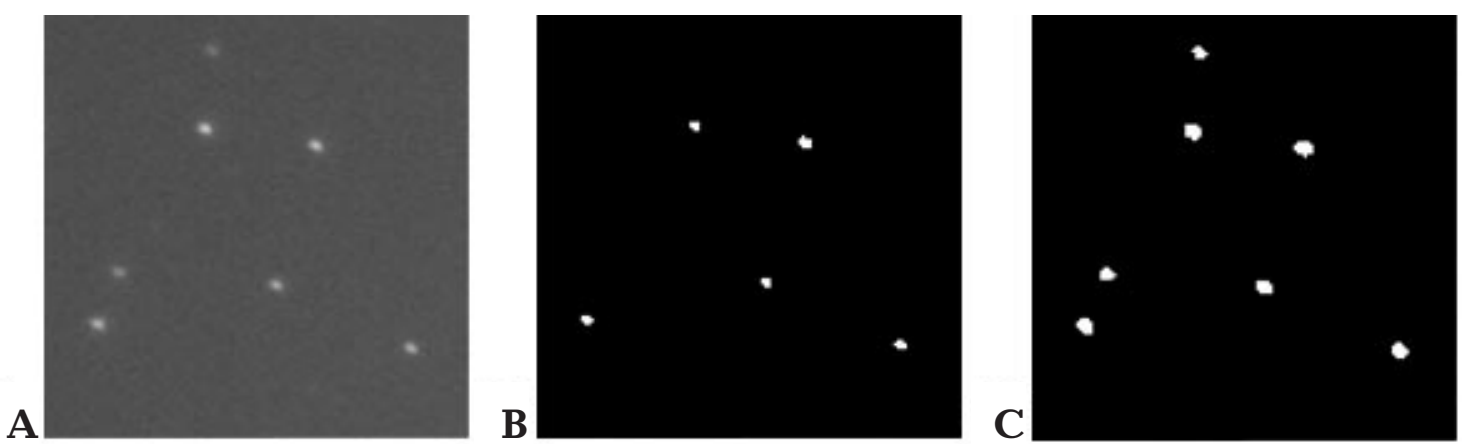

Fig 5. (A) Viruses stained with SYBR Gold. (B,C) Application of the $3 \times 3$ Laplacian filter causes some of the smaller viruses to be filtered out as background noise (B), while the modified $5 \times 5$ Laplacian filter is able to detect the edges of every virus in image (C)

filtering, edge-enhancement, and segmentation adequately under ideal conditions. However, the analysis of images that lack an evenly illuminated background poses a dilemma for segmentation algorithms based around a single global threshold value. The lighting gradient creates an uneven histogram for the image, causing global threshold values to lump large portions of the image into single regions, while to the human eye they are clearly made up of individual objects. The obvious solution to this problem was the development of a segmentation routine that superficially approxi-

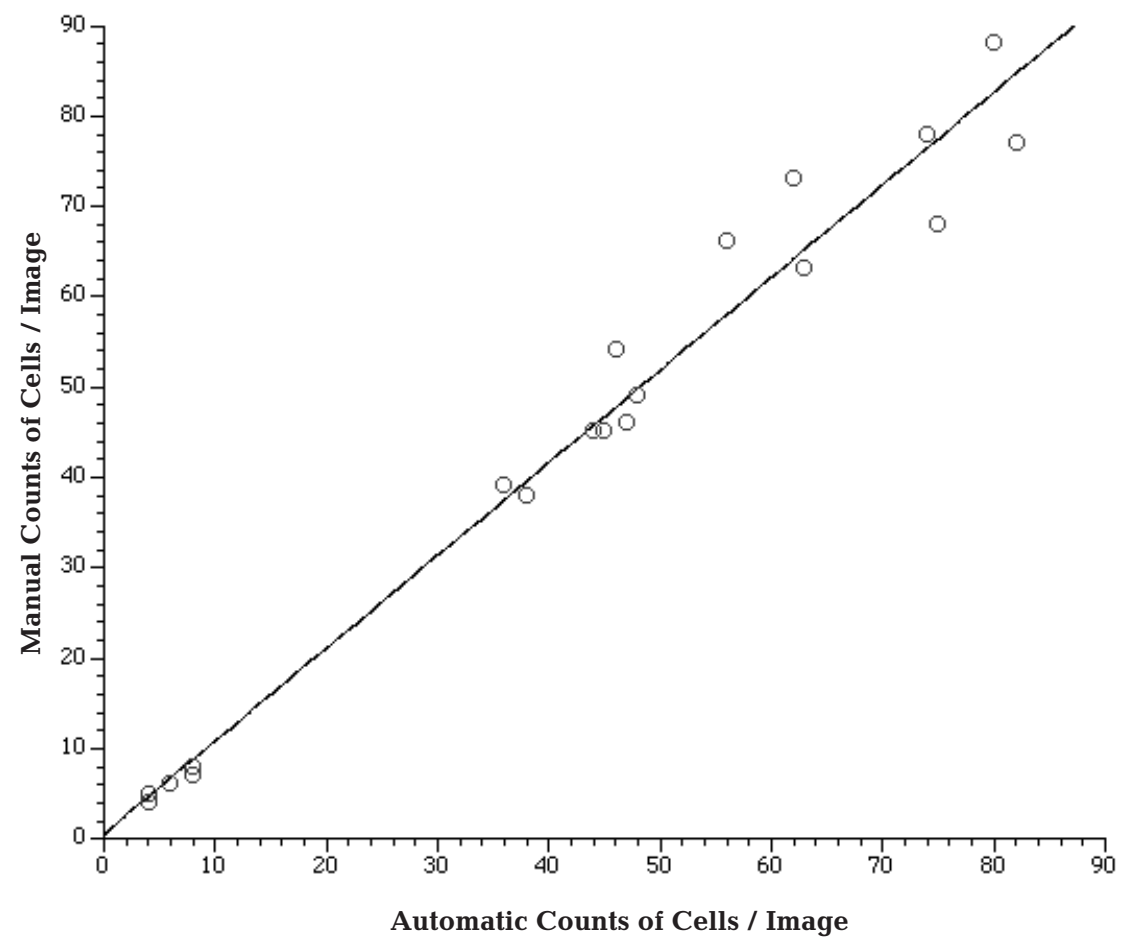

Fig. 6. Linear regression plot of 18 images of bacteria cells enumerated manually and also using the automated 'Skidaway Tools' software. Automated counts $=1.025$ (manual counts) $+0.631 . \mathrm{r}^{2}=0.97, \mathrm{n}=18, \mathrm{p}<0.0001$. SE of intercept $=2.137, \mathrm{SE}$ of slope $=0.042$ mated the edge-detection functions of the human eye. Earlier thresholding and object recognition techniques, such as those described by Sieracki et al. (1989), were based on the Marr-Hildreth edge-detection operator, and used local second derivative intensity to digitally approximate the edge-detection capabilities of natural human vision (Marr \& Hildreth 1980). This method has already been extensively used in both lab (Verity et al. 1992) and field studies (Sieracki et al. 1993, Verity et al. 1994, 1996). This protocol was subsequently updated through the use of the Gaussiansmoothed Laplacian filter routine (Viles \& Sieracki 1991) as a substitute for the direct second-derivative calculations, and has been adapted and modified by a number of image processing packages (Massana et al. 1997, Blackburn et al. 1998). Viles \& Sieracki (1991) also added flat-fielding and edge-strength operators to the solution, thereby providing accurate cell counts under most adverse lighting and noise conditions. While other imaging solutions combine variations on the Laplacian-Gaussian method with other filters and thresholding techniques, the protocol described here maintains the simplicity of the original Laplacian-Gaussian protocol, while taking advantage of the available processing power in contemporary desktop PCs, making it even more efficient for the analysis of images of fluorescently stained cells.

This modified image analysis protocol is capable of generating counts with an accuracy greater than those few commercial image processing packages that contain a global-threshold-based automatic cell counter. The most obvious benefit of procedural image segmenta- 
tion and enumeration is the reduction in operator time necessary to yield accurate results. 'Skidaway Tools', while requiring more processing time than global intensity thresholding systems, does provide a vast improvement over manual counting of multiple digital images. Additionally, the automated nature of the system significantly decreases the potential for human error and bias in an otherwise lengthy enumeration process.
Combining the well-tested and established Gaussian-smoothed Laplacian operator with the detritus masking provides a level of functionality not available previously. Accurate image segmentation protocols are necessary for the analysis of any bacterial sample, and when combined with the ability to discriminate cells associated with detritus, the capability to study natural samples is enhanced and no longer dependent on manual counts of multiple sample images. However,
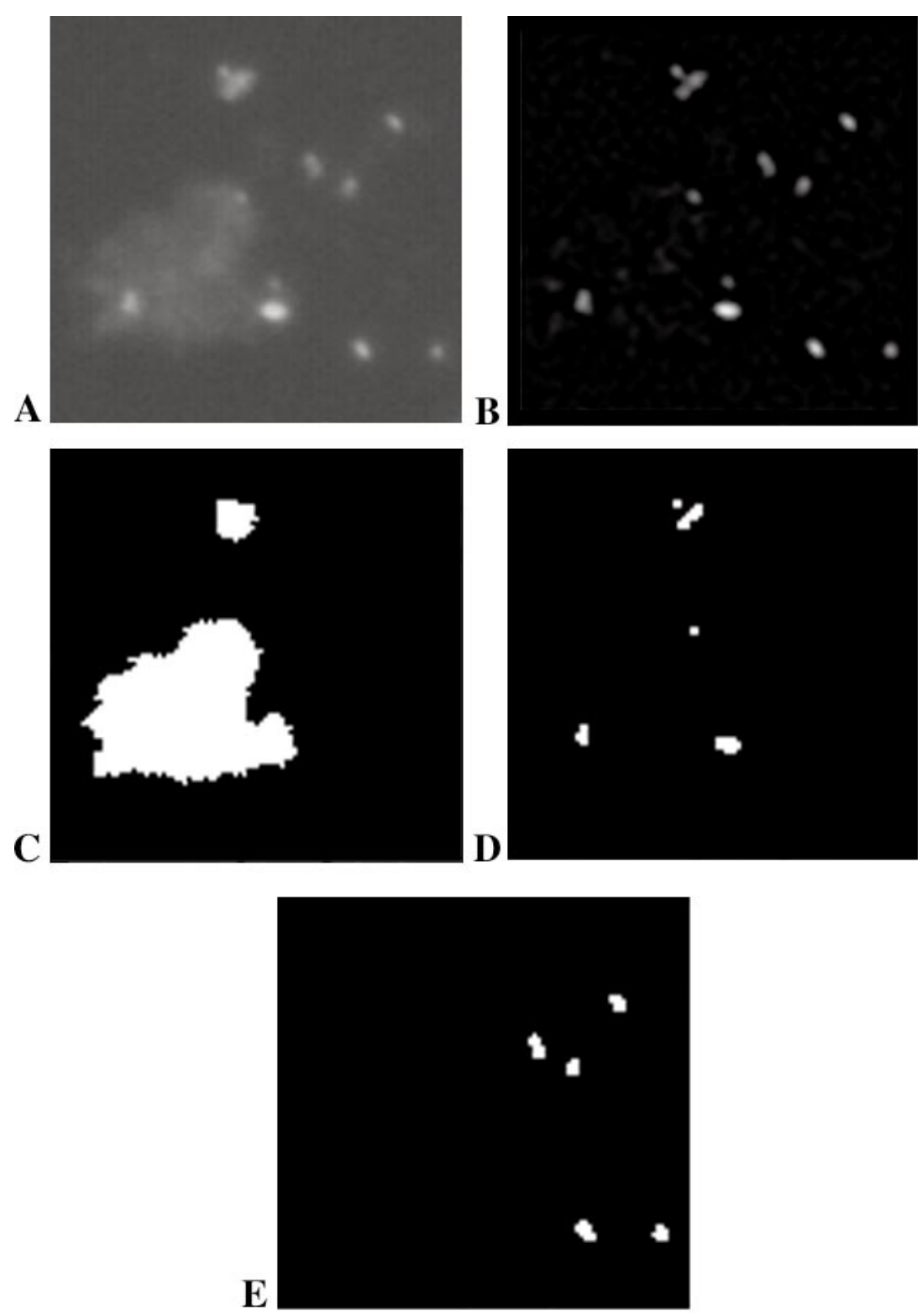

Fig. 7. (A) Estuarine water sample containing detrital matter. (B) Application of the modified $5 \times 5$ Laplacian filter to image (A) results in the detritus being filtered out with the background, while all bacteria cells (free-floating and associated with detritus) are identified. (C) Alpha-channel mask of image (A). (D) The alpha-channel is applied to the segmented image (B), masking out the cells associated with detritus and permitting enumeration of unattached bacteria. (E) The alpha-channel is inverted and reapplied to the original segmented image, masking the background and providing a count of the attached bacteria cells. The edge-strength operator eliminates residual traces of detritus during the segmentation and enumeration process 
improved methods for generating the detrital alphachannel are necessary as the existing method relies too heavily on user input, while the ultimate goal is the development of a fully automated masking system that can be seamlessly integrated into existing segmentation protocols. That notwithstanding, the detrital mask has been shown to be an accurate and effective method for the enumeration of attached and unattached bacteria. This updated segmentation protocol, when applied to VSP (Williams et al. 1998) and other similar fluorescent staining protocols, provides a tool for the precise and efficient analysis of the majority of bacterial samples.

Acknowledgements. The authors wish to thank M. Hauer for assistance with the modified Laplacian filter. D. Peterson prepared the manuscript. This research was supported by National Science Foundation grants OCE-95-21086 and OCE96-17884, and Department of Energy contract no. DOE-FG02-98ER62531.

\section{LITERATURE CITED}

Bjørnsen PK (1986) Automatic determination of bacterioplankton biomass by image analysis. Appl Environ Microbiol 51:1199-1204

Blackburn N, Hagström A, Wikner J, Cuadros-Hansson R, Bjørnsen PK (1998) Rapid determination of bacterial abundance, biovolume, morphology, and growth by neural network-based image analysis. Appl Environ Microbiol 64:3246-3255

Gauthier D, Levine MD, Noble PB (1992) Principles of object detection for an automated cell tracking system. In: Häder DP (ed) Image analysis in biology. CRC Press, Boca Raton, p 9-27

Kepner RL, Pratt JR (1994) Use of fluorochromes for direct enumeration of total bacteria in environmental samples: past and present. Microb Rev 58:603-615

Krambeck K, Krambeck HJ, Overbeck J (1981) Microcomputer-assisted biomass determination of plankton bacteria on scanning electron micrographs. Appl Environ Microbiol 42:142-149

Lu JR, Chen F, Hodson RE (1999) Enumeration of viruses in aquatic environments using SYBR Gold stain and digital image analysis. American Society for Microbiology (ASM) 99th General Meeting Abstracts, Chicago, IL. Abstract N207. ASM, Washington, DC

Editorial responsibility: John Dolan, Edgewater, Maryland, USA
Marr D, Hildreth EC (1980) Theory of edge detection. Proc R Soc Lond B Biol Sci 207:187-217

Massana R, Gasol JM, Bjørnsen PK, Blackburn N, Hagström $\AA$ A, Hietanen S, Hygum BH, Kuparinen J, Pedrós-Alió C (1997) Measurement of bacterial size via image analysis of epifluorescence preparations: description of an inexpensive system and solutions to some of the most common problems. Sci Mar 61:397-407

Schroder D, Krambeck HJ (1991) Advances in digital image analysis of bacterioplankton with epifluorescence microscopy. Verh Int Ver Limnol 24:2601-2604

Sieracki ME, Johnson PW, Sieburth JMcN (1985) Detection, enumeration, and sizing of planktonic bacteria by imageanalyzed epifluorescence microscopy. Appl Environ Microbiol 49:799-810

Sieracki ME, Reichenbach SE, Webb KL (1989) Evaluation of automated threshold selection methods for accurately sizing microscopic fluorescent cells by image analysis. Appl Environ Microbiol 55:2762-2772

Sieracki ME, Verity PG, Stoecker DK (1993) Plankton community response to sequential silicate and nitrate depletion during the 1989 North Atlantic spring bloom. DeepSea Res II 40:213-225

Verity PG, Sieracki ME (1993) Use of color image analysis and epifluorescence microscopy to measure plankton biomass. In: Kemp PF, Sherr BF, Sherr EB, Cole JJ (eds) Handbook of methods in aquatic microbial ecology. Lewis Publishers, Boca Raton, p 327-338

Verity PG, Robertson CY, Tronzo CR, Andrews MG, Nelson JR, Sieracki ME (1992) Relationships between cell volume and the carbon and nitrogen content of marine photosynthetic nanoplankton. Limnol Oceanogr 37:1434-1446

Verity PG, Paffenhöfer GA, Wallace D, Sherr E, Sherr B (1994) Composition and biomass of plankton in spring on the Cape Hatteras shelf, with implications for carbon flux. Cont Shelf Res 16:1087-1116

Verity PG, Stoecker DK, Sieracki ME, Nelson JR (1996) Microzooplankton grazing of primary production at $140^{\circ} \mathrm{W}$ in the equatorial Pacific. Deep-Sea Res II 43: $1227-1255$

Viles CL, Sieracki ME (1991) Measurement of marine picoplankton cell size by using a cooled, charge-coupled device camera with image-analyzed fluorescence microscopy. Appl Environ Microbiol 58:584-592

Williams SC, Verity PG, Beatty T (1995) A new staining technique for dual identification of plankton and detritus in seawater. J Plankton Res 17:2037-2047

Williams SC, Hong Y, Danavall DCA, Howard-Jones MH, Gibson D, Frischer ME, Verity PG (1998) Distinguishing between living and nonliving bacteria: evaluation of the vital stain propidium iodide and its combined use with molecular probes in aquatic samples. J Microbiol Methods 32:225-236

Submitted: December 6, 1999; Accepted: March 10, 2000 Proofs received from author(s): August 10, 2000 